\title{
Decision-making by surgeons about referral for adjuvant therapy for patients with non-small-cell lung, breast or colorectal cancer: a qualitative study
}

\author{
Robin Urquhart PhD, Cynthia Kendell MSc, Gordon Buduhan MD MSc, Daniel Rayson MD, \\ Joan Sargeant PhD, Paul Johnson MD MSc, Eva Grunfeld MD DPhil, Geoffrey A. Porter MD MSc
}

\section{Abstract}

Background: Because surgeons are the main gatekeepers to oncology services, understanding how they make decisions related to referral for adjuvant therapies is important to optimize referral rates and use of oncology services for patients with potentially curable disease. We examined decision-making by surgeons related to referral to oncology services for patients having undergone curativeintent surgery for non-small-cell lung, breast or colorectal cancer.

Methods: We conducted a qualitative study, whose design was guided by the principles of grounded theory. Semi-structured interviews were held with 29 surgeons who performed non-small-cell lung, breast or colorectal cancer surgery in the province of Nova Scotia. Data were collected and analyzed concurrently. Analysis involved an inductive, grounded approach using constant comparative analysis. Data collection and analysis continued until theoretical saturation was reached.

Results: Seven factors influenced the surgeons' decision-making related to referral to oncology services: indications and contraindications for therapy; patients' beliefs and preferences; a belief that oncologists are the experts; knowledge of local standards of care; consultation with oncology colleagues; navigating patient logistics (e.g., lodging, caregiving responsibilities, insurance coverage); and system resources and capacity.

Interpretation: Our study's findings provide a novel understanding of how surgeons make decisions about oncology referral and point to potential areas for intervention to promote referral to oncology services for patients for whom adjuvant therapy is recommended.

\section{I}

ung cancer, breast cancer and colorectal cancer account for $39 \%$ of all new cancer diagnoses in Canada. ${ }^{1}$ These 3 cancers represent the leading causes of cancer-related death, accounting for $46 \%$ of such deaths in $2014 .{ }^{1}$ Early stage non-small-cell lung, breast and colorectal cancers represent potentially curable types of cancer. For patients with these diseases, surgical resection is the primary treatment, with adjuvant therapies (i.e., chemotherapy or radiotherapy, or both) recommended according to the disease stage. Unlike some malignant diseases (e.g., prostate cancer ${ }^{2}$ ), treatment strategies for non-small-cell lung, breast and colorectal cancers are relatively standardized for most patients with localized disease undergoing curative-intent surgery. Based on data from large randomized clinical trials showing improvements in survival outcomes, ${ }^{3-10}$ practice guidelines recommend specific adjuvant therapies for stage II or IIIA non-small-cell lung cancer, stage I-III breast cancer, stage II or III rectal cancer, and stage IIB or III colon cancer. ${ }^{11-16}$ The impact of recommended adjuvant therapies, however, will ultimately depend on their real-world use outside of clinical trial settings.

Across Canada, researchers have observed variations in referral rates and receipt of oncology services for patients with potentially curable cancers. ${ }^{17-21}$ Prior research in the province of Nova Scotia showed that a substantial minority of patients with potentially curable non-small-cell lung or colorectal cancer $(20 \%-33 \%$, depending on cancer site and stage) were not referred for an oncology consultation. ${ }^{17,21}$ For solid tumours, surgeons are the main gatekeepers to adjuvant therapy ser-

Competing interests: None declared.

This article has been peer reviewed.

Correspondence to: Robin Urquhart, robin.urquhart@nshealth.ca CMAJ Open 2016. DOI:10.9778/cmajo.20150030 
vices. Although some patients are not referred because of age or comorbidity, there may be other factors that influence the decision not to refer. We undertook this study to explore how cancer surgeons make decisions related to oncology referral. We examined decision-making by surgeons related to referral to oncology services for patients having undergone curativeintent surgery for non-small-cell lung, breast and colorectal cancer. The resulting knowledge was deemed important to inform the development of appropriate interventions to optimize referral rates and utilization of oncology services for patients with potentially curable disease.

\section{Methods}

\section{Study design}

For this qualitative study, we used grounded theory methodol$\mathrm{ogy}^{22}$ with semi-structured interviews of cancer surgeons. Qualitative inquiry permits exploration of the breadth of factors influencing health care decisions and the complexity behind health care decision-making..$^{23,24}$ Grounded theory attempts to move beyond description and generate a general explanation, or theory, of a process or action that is shaped by the views of participants who have experienced the process or action. ${ }^{22}$

The study was informed by our ongoing research as well as the Penchansky and Thomas model of access to health care services (Table 1). ${ }^{25}$ The 6 dimensions described in this model guided data collection and analyses.

Ethical approval to conduct the study was obtained from the research ethics boards in the province's 10 health regions. Written informed consent was obtained from each participant; this included permission to audiotape the interviews and use anonymized quotes.

\section{Participants}

Participants were surgeons in the province of Nova Scotia who were performing non-small-cell lung, breast or colorectal can- cer surgery. The province has a population of about 940000 . During this study, 60 surgeons were performing lung, breast or colorectal cancer surgery in the province. Surgeons were purposively recruited, with 2 investigators (R.U. and G.A.P.) identifying all potential participants to ensure variation in career stage (junior and senior), level of training (general surgeons and surgical oncologists) and practice location (community hospitals and academic/tertiary care centres). A research coordinator initially approached each potential participant via email or telephone to introduce the study and invite him or her to participate. Those who failed to respond were approached again within a week. If the participant responded in the affirmative, the research coordinator scheduled a time to obtain informed consent and conduct the interview.

\section{Data collection}

Semistructured interviews were held in person in the surgeon's office or via telephone, depending on practical considerations. Questions and related probes were drafted based on the research objective, team members' clinical experiences, and the Penchanksy and Thomas model. (The interview guide is available in Appendix 1, www.cmajopen.ca/content/4/1/E7/ suppl/DC1.) Two pilot interviews were conducted to assess and refine the interview script; these were audiotaped, transcribed verbatim and discussed among the entire research team to ensure that all topics of interest were explored.

One investigator (R.U.), with expertise in qualitative methods, conducted all of the interviews. Only the investigator and interviewee were involved in the interview. The investigator had prior understanding of the work of the participants; ensured participants understood the study objectives, the reason for doing the study (i.e., prior research had shown suboptimal referral practices) and the interview procedure; and encouraged participants to express their opinions by explaining that all responses were valid and valuable and would be included in the analysis. Most participants practising in

Table 1: Six dimensions related to the Penchansky and Thomas model of access to health care services ${ }^{25}$

\begin{tabular}{|c|c|c|}
\hline Model & Dimension & Examples \\
\hline \multirow{5}{*}{$\begin{array}{l}\text { Original Penchansky } \\
\text { and Thomas model }\end{array}$} & Availability of health care resources & Resources (personnel, equipment, technology), prevailing wait times \\
\hline & $\begin{array}{l}\text { Accessibility as it relates to geographic } \\
\text { considerations }\end{array}$ & Centralized services, "close to home" care, transportation \\
\hline & $\begin{array}{l}\text { Accommodation in terms of how health } \\
\text { care is organized and delivered }\end{array}$ & $\begin{array}{l}\text { Coordination and integration of services, satellite cancer clinics, } \\
\text { telemedicine }\end{array}$ \\
\hline & $\begin{array}{l}\text { Affordability as it relates to direct and } \\
\text { indirect costs of receiving care }\end{array}$ & $\begin{array}{l}\text { Funding of cancer services, insurance/drug coverage, indirect patient } \\
\text { costs (lodging, transportation) }\end{array}$ \\
\hline & $\begin{array}{l}\text { Acceptability as it relates to the attitudes } \\
\text { and characteristics of patients and } \\
\text { providers }\end{array}$ & $\begin{array}{l}\text { Patient and provider attitudes toward one another, patient } \\
\text { characteristics (e.g., age, sex, comorbid conditions, life expectancy), } \\
\text { patient preferences, provider characteristics (sex, years of practice, } \\
\text { level of specialization, surgery volume) }\end{array}$ \\
\hline Added $^{*}$ & $\begin{array}{l}\text { Awareness of services and indications } \\
\text { for their use }\end{array}$ & $\begin{array}{l}\text { Patient and provider awareness of evidence for therapy, clinical } \\
\text { practice guidelines, structures that support multidisciplinary dialogue/ } \\
\text { consultation }\end{array}$ \\
\hline
\end{tabular}


academic/tertiary care centres knew the investigator before the study, whereas most practising in community hospitals did not. All of the interviews were audiotaped, transcribed verbatim by a research coordinator with experience in transcription and verified by the investigator by listening to the audiotapes. The audiotapes and transcripts were supplemented with field notes. Consistent with grounded theory, the interview guide was adapted during data collection on the basis of previous interview findings to further explore important concepts and emerging categories. ${ }^{27,28}$

\section{Analysis}

Data were collected and analyzed concurrently. For the analysis, we used an inductive, grounded approach and constant comparative analysis. ${ }^{22}$ The pilot interviews were included in the analysis, with pilot participants' permission.

Data from the first 14 interviews (about half) were coded and analyzed independently by 2 investigators (R.U. and C.K.). Through iterative discussion, they developed a code book, which reflected unique ideas and concepts, to guide the coding scheme and subsequent categorization of data. Consistent with constant comparative analysis, open and axial coding of interview transcripts occurred simultaneously. These processes involved reading and rereading of transcripts, applying the coding scheme to the interview text and grouping the coded text into more abstract categories. Qualitative analysis was performed manually, with the assistance of qualitative software (NVivo; QSR International) for data management and to enable comparison and synthesis of codes.

Because consistency in coding was evident, the remaining 15 interview transcripts were coded and analyzed by 1 investigator (C.K.), with regular meetings between both investigators (R.U. and C.K.) to review the coded data, discuss emerging categories and probe theoretical saturation. The field notes helped inform these discussions. Selective coding, or the detailed development of categories, selection of a core theoretical category and integration of categories, was performed through discussion between both investigators. Disagreements were resolved through discussion and, when needed, reexamination of interview transcripts and coded data.

Three in-person team meetings were held to discuss and question emerging categories, confirm categories, and discuss and question the organization of categories into a coherent explanation. The in-person meetings were supplemented by email discussions. These processes were iterative, and they continued until team members perceived that the final categories and their organization adequately captured and reflected the findings. Data collection and analysis continued until theoretical saturation was reached (i.e., the point whereby no new substantive information was being collected to develop the explanation). ${ }^{22}$

Numerous steps were taken to optimize the rigour of this study. These included field notes during interviews; detailed documentation of methodologic and analytic decisions; systematic data coding; use of direct quotations to ensure participant perspectives were represented as clearly as possible; ongoing review and questioning of data coding, analytic deci- sions and resultant categories by 2 investigators (R.U. and C.K.); 3 team meetings to discuss and question findings; and provision of a summary of the preliminary findings to the participants. The team members included 3 surgeons (G.A.P., P.J. and G.B.), a medical oncologist (D.R.) and an experienced grounded theorist (J.S.).

\section{Results}

Of 33 surgeons contacted, 29 participated in the study. Two declined participation, and 2 agreed to participate but their interviews had not been scheduled by the time theoretical saturation was reached and data collection was stopped. Interviews lasted between 20 and 49 minutes; $38 \%$ were held in person. There were no repeat interviews. Of the 29 participants, 24 (33\%) were male, 17 (59\%) practised in a community hospital, and 7 (24\%), 8 (28\%) and 14 (48\%) had been practising for less than 5 years, $5-15$ years and 16 or more years, respectively.

Seven factors were found to influence surgeon decisionmaking related to oncology referral, with the degree of influence depending on their decisional proximity (Figure 1). Each factor is briefly described in Box 1. At the core of surgeon decision-making is the clinical encounter wherein the decision is made. Within this encounter, surgeons consider and negotiate their understanding of indications and contraindications for adjuvant therapy (e.g., tumour pathology, patient health status) alongside patient beliefs and preferences (e.g., the desire or not for chemotherapy). These factors are illustrated by the following comments from participants:

You can be 60 and too frail, you know? Sixty and really have the frailty of most of my 90 -year-olds. ... In my opinion these are patients that, you know, the oncologist doesn't necessarily need to see. [Surgeon 01]

When you break it down, some will digest that and say "thanks, but no thanks," but others, they want to be more aggressive and will opt for it. ... [If] they just don't want it and are not interested, then I won't [refer]. [Surgeon 08]

Germane to the referral decision are a number of important mediating factors. One of these is a belief that oncologists are the experts, which facilitates referral recommendations:

What I tell patients is that "I am not the expert on this, see what they have to say." So I don't want to prevent them from getting a therapy because $[\mathrm{I} \mathrm{am}]$ a gatekeeper. If you don't refer them, they don't get it. [Surgeon 09]

Other mediating factors include knowledge of local standards of care and consultation with oncology colleagues. These 2 factors reflect communication and integration mechanisms (or lack thereof) between surgeons and the formal oncology programs, as illustrated in the following comments:

[T] he biggest problem is the oncologists in [centre $\mathrm{A}]$ do not communicate with those of us outside of [centre A] very well in what 
their feelings are on management and that sort of thing. When new studies are published and they change their practice, they don't tell the rest of us. ... I ask them "well, what is the group's approach to this?" and they don't communicate. [Surgeon 20]

The benefit of having people you can call is for the people that don't fit the mold, and you can't plug everybody into the same protocol and you can't plug everybody into the same algorithm, and when you have got those people, it is wonderful to call [a colleague] and say, "this is not the run of the mill." [Surgeon 26]

When making decisions about oncology referral, surgeons are also acutely aware of the outer context in which these decisions occur, including system resources and capacity (e.g., access to staging investigations) and a need to navigate patient logistics (e.g., drug coverage, and transportation and lodging). Although factors in this outer context infrequently influence referral decisions directly, they often make dealing with the referral more difficult, as described below:

It is quite a slog to get a patient navigated through the system quickly, and truthfully it is hit and miss. ... I have got a patient who, by standard of care and guidelines, I can't operate on [because he/she has not received a staging MRI] and who has symptoms and needs treatment but also needs the emotional reassurance that things are underway and they are getting something. [Surgeon 01]

We try to use our social service people as much as possible and we try and engage other family members, community organizations. ... In our rural areas, we have some really good volunteer groups that look after cancer patients. ... You have to deal with the situation that you have and try to give every patient the best opportunity to get the best treatment available, and sometimes that requires jumping through extra hoops. [Surgeon 07]

\section{Interpretation}

This study examined surgeon decision-making regarding referral to oncology for patients following curative-intent surgery for non-small-cell lung, breast or colorectal cancer. The findings show that surgeons' understandings of indications and contraindications for adjuvant therapy and patients' beliefs and preferences clearly influence whether a patient is referred to oncology services. These 2 factors reflect a decision-making process largely aligned with the principles of evidence-based medicine. ${ }^{29-31}$ Other factors influencing referral decisions included surgeons' respect for oncologists' knowledge and

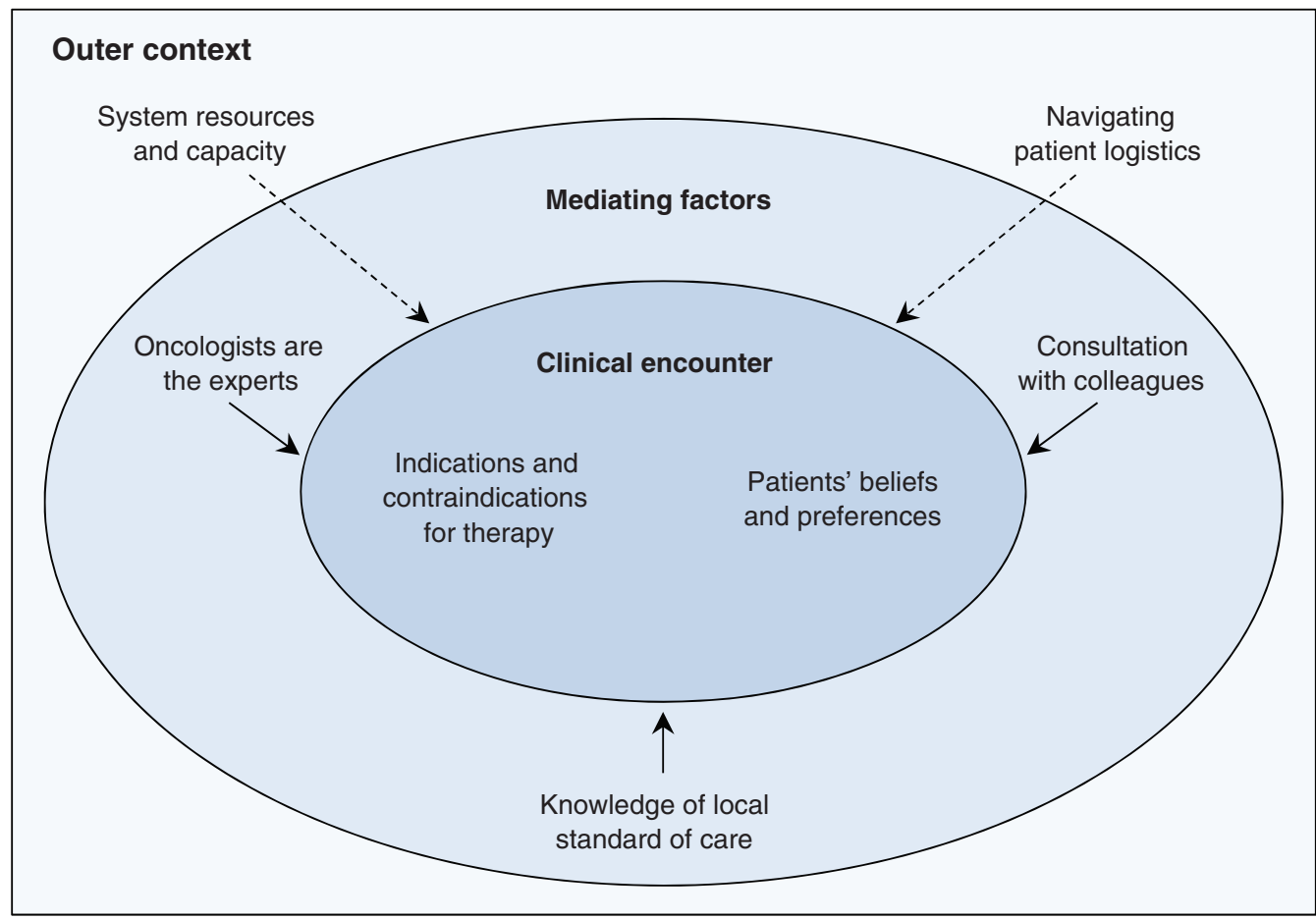

Figure 1: Factors influencing surgeons' decision-making related to referral of patients with potentially curable cancer (non-small-cell lung, breast or colorectal cancer) to oncology services for consideration of adjuvant therapy. The degree of influence depends on decisional proximity. 
expertise, awareness of local standards of care and relationships with oncology colleagues. Together, these findings provide important and novel insights into the decision-making process related to oncology referral, and thus offer potential target areas for intervention.

That surgeons reported uncertainty about local standards of care and discussed informally consulting colleagues about referral decisions, in the absence of accessible communication mechanisms, are important to consider in the context of implementing evidence-based medicine. Prior research has shown that surgeons and oncologists have conflicting views on guideline recommendations for adjuvant therapies for breast ${ }^{32}$ and colorectal cancer, ${ }^{33}$ and that preferred approaches for the adjuvant management of non-small-cell lung cancer vary widely within and across medical and surgical specialties. ${ }^{34}$ Thus, clinicians may have varied perspectives on the benefits and risks of cancer treatment and different interpretations of an increasingly complex evidence base. This implies that clarity on local management protocols and standards as well as opportunities to discuss management options are of great importance.

Surgeons in this study also highlighted the importance of logistical supports when referring patients to oncologists. In Nova Scotia, receipt of an oncology consultation requires most patients to travel to a cancer centre. For some patients, this involves significant out-of-pocket costs, transportation challenges, and disruptions at home and work. Although these factors may not directly affect the decision to refer, they do change the clinical encounter and pose considerable challenges for some patients (e.g., those with limited resources or with dependents at home). Many researchers have shown that the financial burden associated with cancer is often severe across Canadian jurisdictions. ${ }^{35-39}$

\section{Limitations}

The main limitation of this study is that it occurred in a single province, potentially limiting generalizability. The purpose of qualitative research, however, is not to achieve generalizable results but to acquire detailed knowledge about processes and context, and underlying causal mechanisms (i.e., the why and how). This study included many steps to maximize rigour, had a high participation rate, attained theoretical saturation and observed commonalities across disease sites. As such, these findings should have applicability to other similar settings (e.g., publicly funded health care, centralized oncology services, or settings where surgeons are the main gatekeeper to oncology services).

\section{Conclusion}

These findings provide a novel understanding of how surgeons make decisions about oncology referral and point to potential areas for intervention to promote referral to oncology services for patients for whom adjuvant therapy is recommended. These areas include development and implementation of provincial standards, improvements in access to multidisciplinary tumour boards and the development of systems that permit "just-in-time" surgeon-oncologist consultation. Such interventions can increase collaborative decision-making ${ }^{21}$ and support co-management options for community-based surgeons who do not regularly interact with other cancer specialists. ${ }^{40}$ Policy interventions that provide supportive and financial supports to

Box 1: Factors influencing surgeons' decision-making related to referral of patients to oncology services

\section{Clinical encounter}

- Indications and contraindications for therapy: Surgeons consider indications and contraindications based on tumour characteristics (e.g., lymph node status, stage, high-risk features), length of time after surgery, and the patient's age and health status (e.g., frailty, comorbidities).

- Patients' beliefs and preferences:* Surgeons' decisions are influenced by patients' beliefs and preferences. If a patient desires a consultation, he or she is normally referred. If a patient is reluctant to see a medical or radiation oncologist or expresses fear with regard to adjuvant therapy but the surgeon feels a consultation is warranted, the surgeon will attempt to convince the patient to have the consultation but will recognize that it is the patient's choice.

\section{Mediating factors}

- Knowledge of local standards of care:* Surgeons' decisions are influenced by their knowledge (or lack thereof) of local standards of care. Surgeons reported mixed messages from oncologists and confusion about whether to refer certain patients. This was particularly true for community surgeons.

- Consultation with colleagues:* Surgeons perceive formal processes to consult with colleagues to be largely lacking or inaccessible. They rely on relationships with colleagues and believe their informal consultations with these colleagues help their decisions and improve patient care.

- Oncologists are the experts:† Surgeons' decisions are influenced by their belief that medical and radiation oncologists are experts in adjuvant therapy and thus better able to address patients' fears or concerns and need for information, and to advise on contraindications for adjuvant therapy and potential adverse effects.

\section{Outer context}

- Navigating patient logistics: $¥$ Surgeons discussed many logistical factors (e.g., lodging, caregiving responsibilities, insurance coverage) that influence a patient's willingness or ability to consult an oncologist. Typically, if a surgeon deems a referral appropriate and is aware that logistical factors are an issue, the surgeon refers the patient but simultaneously attempts to access supports on the patient's behalf. Surgeons varied greatly with regard to their awareness of available resources for patients.

- System resources and capacity:§ Surgeons are aware of the limitations of the health care system (e.g., suboptimal access to investigations, shortage of human resources, wait times and inadequate technology systems). These limitations tend not to affect decision-making directly, but they can complicate referral processes and affect patients' timely access to oncology services.

*This factor encompasses data from $100 \%$ of study participants. †This factor encompasses data from $97 \%$ of study participants. $\ddagger$ This factor encompasses data from $97 \%$ of study participants. However, 5 of these participants, when asked, stated this factor does not directly influence their referral decisions, but they described dealing with or managing patient logistics upon referral.

$\S$ This factor encompasses data from $97 \%$ of study participants. However, 7 of these participants, when asked, stated this factor does not directly influence their referral decisions, but they described dealing with or managing system resource and capacity issues upon referral. 
improve patient access to oncology are also needed. Future work should focus on designing and testing such interventions, in collaboration with surgeons and their clinical colleagues, to understand which ones work, in which settings and why.

\section{References}

1. Advisory Committee on Cancer Statistics. Canadian cancer statistics 2014. Toronto: Canadian Cancer Society; 2014.

2. Birnie K, Robinson J. Helping patients with localized prostate cancer reach treatment decisions. Can Fam Physician 2010;56:137-41.

3. Arriagada R, Bergman B, Dunant A, et al. Cisplatin-based adjuvant chemotherapy in patients with completely resected non-small-cell lung cancer. $N$ Engl $7 \mathrm{Med}$ 2004;350:351-60.

4. Douillard JY, Rosell R, De Lena M, et al. Adjuvant vinorelbine plus cisplatin versus observation in patients with completely resected stage IB-IIIA nonsmall-cell lung cancer cancer (Adjuvant Navelbine International Trialist Association [ANITA]): a randomised controlled trial. Lancet Oncol 2006;7:719-27.

5. Early Breast Cancer Trialists' Collaborative Group. Effects of chemotherapy and hormonal therapy for early breast cancer on recurrence and 15-year survival: an overview of the randomised trials. Lancet 2005;365:1687-717.

6. Gray R, Barnwell J, McConkey C, et al.; QUASAR Collaborative Group. Adjuvant chemotherapy versus observation in patients with colorectal cancer: a randomised study. Lancet 2007;370:2020-9.

7. Sargent DJ, Goldberg RM, Jacobson SD, et al. A pooled analysis of adjuvant chemotherapy for resected colon cancer in elderly patients. N Engl 7 Med 2001; 345:1091-7.

8. Strauss GM, Herndon J, Maddaus MA, et al. Randomized clinical trial of adjuvant chemotherapy with paclitaxel and carboplatin following resection in stage IB non-small cell lung cancer (NSCLC): report of Cancer and Leukemia Group B (CALGB) Protocol 9633. 7 Clin Oncol 2004;22(14 Suppl):7019.

9. Winton T, Livington R, Johnson D, et al. Vinorelbine plus cisplatin vs. observation in resected non-small-cell lung cancer. N Engl 7 Med 2005;352:2589-97.

10. Wolpin BM, Meyerhardt JA, Mamon HJ, et al. Adjuvant treatment of colorectal cancer. CA Cancer 7 Clin 2007;57:168-85.

11. Breast Cancer Evidence-based Series (EBS) and Practice Guidelines (PG). Toronto: Cancer Care Ontario; 2011.

12. Gastrointestinal Cancer Evidence-based Series (EBS) and Practice Guidelines (PG). Toronto: Cancer Care Ontario; 2011.

13. Lung Cancer Evidence-based Series (EBS) and Practice Guidelines (PG). Toronto: Cancer Care Ontario; 2011.

14. Figueredo A, Fine S, Maroun J, et al. Adjuvant therapy for stage III colon cancer after complete resection. Provincial Gastrointestinal Disease Site Group. Cancer Prev Control 1997;1:304-9.

15. Pisters KM, Evans WK, Azzoli CG, et al. Cancer Care Ontario and American Society of Clinical Oncology adjuvant chemotherapy and adjuvant radiation therapy for stages I-IIIA resectable non small-cell lung cancer guideline. 7 Clin Oncol 2007;25:5506-18.

16. Wong R, Berry S, Spithoff K, et al. Preoperative or postoperative therapy for the management of patients with stage II or III rectal cancer: guideline recommendations. Evidence-Based Series \#2-4. Toronto: Cancer Care Ontario; 2008.

17. Younis T, Al-Fayea T, Virik K, et al. Adjuvant chemotherapy uptake in nonsmall cell lung cancer. 7 Thorac Oncol 2008;3:1272-8.

18. Ong S, Watters JM, Grunfeld E, et al. Predictors of referral for adjuvant therapy for colorectal cancer. Can 7 Surg 2005;48:225-9.

19. Winget M, Hossain S, Yasui Y, et al. Characteristics of patients with stage III colon adenocarcinoma who fail to receive guideline-recommended treatment. Cancer 2010;116:4849-56.

20. Winget M, Stanger T, Gao Z, et al. Predictors of surgery and consult with an oncologist for adjuvant chemotherapy in early stage NSCLC patients in Alberta, Canada. 7 Thorac Oncol 2009;4:629-34.

21. Rayson D, Urquhart R, Cox M, et al. Adherence to clinical practice guidelines for adjuvant chemotherapy for colorectal cancer in a Canadian province: a populationbased analysis. 7 Oncol Pract 2012;8:253-9.

22. Strauss AL, Corbin JM. Basics of qualitative research: grounded theory procedures and techniques. Thousand Oaks (CA): Sage; 1990.

23. Williams I, Bryan S. Understanding the limited impact of economic evaluation in health care resource allocation: a conceptual framework. Health Policy 2007; $80: 135-43$.

24. Freemantle N, Mason J. Not playing with a full DEC: why development and evaluation committee methods for appraising new drugs may be inadequate. BMF 1999;318:1480-2
25. Penchansky R, Thomas JW. The concept of access: definition and relationship to consumer satisfaction. Med Care 1981;19:127-40.

26. Mackillop WJ. Health services research in radiation oncology: towards achieving the achievable for patients with cancer. In: Gunderson LL, Tepper JE, editors. Clinical radiation oncology. 2nd ed. Philadelphia (PA): Churchill Livingstone; 2006.

27. Patton MQ. Qualitative research \& evaluation methods. 3rd ed. Thousand Oaks (CA): Sage Publications; 2002.

28. Rubin H, Rubin I. Qualitative interviewing: the art of hearing data. Thousand Oaks (CA): Sage Publications; 1995.

29. Haynes RB, Devereaux PJ, Guyatt GH. Physicians' and patients' choices in evidence based practice. BM7 2002;324:1350.

30. Haynes RB, Sackett DL, Gray JM, et al. Transferring evidence from research into practice: 1 . The role of clinical care research evidence in clinical decisions. ACP 7 Club 1996;125:A14-6.

31. Sackett DL, Rosenberg WM, Gray JA, et al. Evidence based medicine: What it is and what it isn't. BM7 1996;312:71-2.

32. Jagsi R, Abrahamse P, Morrow M, et al. Coordination of breast cancer care between radiation oncologists and surgeons: a survey study. Int 7 Radiat Oncol Biol Phys 2012;82:2072-8.

33. Keating NL, Landrum MB, Klabunde CN, et al. Adjuvant chemotherapy for stage III colon cancer: Do physicians agree about the importance of patient age and comorbidity? 7 Clin Oncol 2008;26:2532-7.

34. Schroen AT, Detterbeck FC, Crawford R, et al. Beliefs among pulmonologists and thoracic surgeons in the therapeutic approach to non-small cell lung cancer. Chest 2000;118:129-37.

35. Grunfeld E, Coyle D, Whelan T, et al. Family caregiver burden: results of longitudinal study of breast cancer patients and their principal caregivers. CMA7 2004:170:1795-802.

36. Longo CJ, Fitch M, Deber RB, et al. Financial and family burden associated with cancer treatment in Ontario, Canada. Support Care Cancer 2006;14:1077-85.

37. Longo CJ, Deber R, Fitch $M$, et al. An examination of cancer patients' monthly 'out-of-pocket' costs in Ontario, Canada. Eur 7 Cancer Care (Engl) 2007;16:500-7.

38. Lauzier S, Maunsell E, Drolet M, et al. Wage losses in the year after breast cancer: extent and determinants among Canadian women. 7 Natl Cancer Inst 2008;100:321-32.

39. Mathews M, West R, Buehler S. How important are out-of-pocket costs to rural patients' cancer care decisions? Can 7 Rural Med 2009; 14:54-60.

40. Rogers SO Jr, Ayanian JZ, Ko CY, et al. Surgeons' volume of colorectal cancer procedures and collaborative decision-making about adjuvant therapies. Ann Surg 2009;250:895-900.

Affiliations: Department of Surgery (Urquhart, Johnson, Porter), Department of Community Health and Epidemiology (Urquhart, Johnson, Porter), Division of Medical Education (Sargeant) and Division of Medical Oncology (Rayson), Dalhousie University, Halifax, NS; Cancer Outcomes Research Program (Urquhart, Kendell, Porter), Capital District Health Authority/Dalhousie University, Halifax, NS; Department of Surgery (Buduhan), University of Manitoba, Winnipeg, Man.; Ontario Institute for Cancer Research (Grunfeld), Toronto, Ont.; Department of Family and Community Medicine (Grunfeld), University of Toronto, Toronto, Ont

Contributors: Robin Urquhart led the conception, design, conduct, analysis and interpretation of the study. The remaining authors contributed to the study design, and the analysis and interpretation of data. Robin Urquhart drafted the article. All of the authors revised the article critically for intellectual content, gave final approval of the version to be published and agreed to act as guarantors of the work.

Funding: This study was funded by the Canadian Institutes of Health Research (grant no. MOP-119493). The funder had no role in the design of the study; the collection, analysis or interpretation of the data; the writing of the manuscript; or the decision to submit the manuscript for publication.

Acknowledgement: The authors gratefully acknowledge Margaret Jorgensen for her assistance with study coordination.

Supplemental information: For reviewer comments and the original submission of this manuscript, see www.cmajopen.ca/content/4/1/E7/ suppl/DC1 